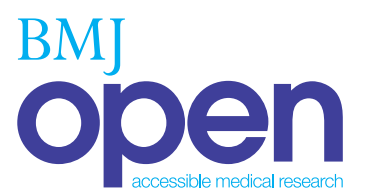

\title{
CoMPASs: IOn programme (Care Of Memory Problems in Advanced Stages of dementia: Improving Our Knowledge): protocol for a mixed methods study
}

To cite: Jones L, Harrington J, Scott S, et al. CoMPASs: IOn programme (Care Of Memory Problems in Advanced Stages of dementia: Improving Our Knowledge): protocol for a mixed methods study. BMJ Open 2012;2: e002265. doi:10.1136/ bmjopen-2012-002265

- Prepublication history for this paper are available online. To view these files please visit the journal online (http://dx.doi.org/10.1136/ bmjopen-2012-002265)

Received 25 October 2012 Accepted 30 October 2012

This final article is available for use under the terms of the Creative Commons Attribution Non-Commercial 2.0 Licence; see http://bmjopen.bmj.com

Marie Curie Palliative Care Research Unit, UCL Mental Health Sciences Unit, University College London Medical School, London, UK

\section{Correspondence to} Dr Elizabeth L Sampson; e.sampson@ucl.ac.uk

\section{ABSTRACT}

Introduction: Approximately 700000 people in the UK have dementia, rising to 1.2 million by 2050; onethird of people aged over 65 will die with dementia. Good end-of-life care is often neglected, and detailed UK-based research on symptom burden and needs is lacking. Our project examines these issues from multiple perspectives using a rigorous and innovative design, collecting data which will inform the development of pragmatic interventions to improve care.

Methods and analysis: To define in detail symptom burden, service provision and factors affecting care pathways we shall use mixed methods: prospective cohort studies of people with advanced dementia and their carers; workshops and interactive interviews with health professionals and carers, and a workshop with people with early stage dementia. Interim analyses of cohort data will inform new scenarios for workshops and interviews. Final analysis will include cohort demographics, the symptom burden and health service use over the follow-up period. We shall explore the level and nature of unmet needs, describing how comfort and quality of life change over time and differences between those living in care homes and those remaining in their own homes. Data from workshops and interviews will be analysed for thematic content assisted by textual grouping software. Findings will inform the development of a complex intervention in the next phase of the research programme.

Ethics and dissemination: Ethical approval was granted by National Health Service ethical committees for studies involving people with dementia and carers (REC refs. 12/EE/0003; 12/L0/0346), and by university ethics committee for work with healthcare professionals (REC ref. 3578/001). We shall present our findings at conferences, and in peer-reviewed journals, prepare detailed reports for organisations involved with end-of-life care and dementia, publicising results on the Marie Curie website. A summary of the research will be provided to participants if requested.

\section{ARTICLE SUMMARY}

Article focus

- This article describes a mixed methods study to gain an in-depth understanding of the end-of-life experience of people with advanced dementia and their carers.

Key messages

- By 2050, it is anticipated that one-third of people aged over 65 will die with dementia.

- This research will provide evidence from multiple perspectives on the main components of good end-of-life care that are currently often neglected in dementia.

Strengths and limitations of this study

- To our knowledge the quantitative data from the cohort studies will be the first of this nature to be collected in the UK

- The workshops and interactive interviews will provide richness and background to this quantitative data, allowing it to be placed in the context of a complex health and social care system.

- This research does not address the palliative and end-of-life needs of those dying at earlier stages of dementia from other comorbid conditions.

\section{INTRODUCTION}

Approximately 700000 people in the UK have dementia. This will rise to around 840000 by 2026 and 1.2 million by $2050 .{ }^{1}$ One-third of people aged over 65 in the UK will die while suffering from dementia. ${ }^{2}$ In 2005 , it was estimated that 24.3 million people worldwide had dementia with 4.6 million new cases occurring every year, mostly in developing countries. People with dementia have significantly increased death rates ${ }^{3}$ and even minor cognitive impairment is a strong independent predictor of mortality. ${ }^{4}$ Dementia typically involves a long, gradual decline in cognition, behaviour and function. In this research we consider 
people in the advanced stages of this disease who are approaching death.

\section{Challenges}

Essential components of good end-of-life care are often neglected in dementia, and referral to palliative care is rare. ${ }^{5}$ Fewer than $1 \%$ of hospice patients in Europe have a neurological diagnosis. ${ }^{6}$ Despite concerns about prognostic uncertainty, behavioural problems or communication difficulties, ${ }^{78}$ most symptoms such as pain or difficulties swallowing could be managed with good generalist care. ${ }^{9}$ Providing care at home is an aim of the UK Government's End of Life Care Strategy; while benefitting patients and carers it aims to save National Health Service (NHS) costs by avoiding acute hospital admissions. ${ }^{9}$ A recent National Audit Office report indicated that about $50 \%$ of care home residents who died in hospital could have died at home. ${ }^{10}$ In dementia, research has tended to emphasise specific interventions such as pain control, or the withdrawal of care, for example, not prescribing antibiotics or withdrawing feeding. ${ }^{11-15}$ However, good care may require a more rounded (but cost effective) palliative approach, tailored individually to address patient symptoms and the needs of informal carers, particularly in the terminal phase and in bereavement.

\section{Clinical picture}

Advanced dementia can be defined using the Functional Assessment Staging Scale (FAST) level 7 and above ${ }^{16}$ : the person cannot dress themselves, is doubly incontinent and speaks only a few words. With a median survival of 1.3 years, life expectancy is similar to metastatic breast cancer. ${ }^{17} \mathrm{~A}$ retrospective UK study of the last year of life showed that symptom burden and care needs were comparable to those in cancer; in particular $64 \%$ of dementia patients experienced pain (compared with 59\% with cancer), dyspnoea $(46 \%)$, pressure sores $(39 \%)$ and difficulty swallowing or loss of appetite $(86 \%) .{ }^{17}{ }^{18}$ However, detailed research to define the symptom burden and needs of people with advanced dementia at the end-of-life and their informal carers has not been conducted in the UK.

\section{Care Of Memory Problems in Advanced Stages: Improving Our Knowledge programme}

In this paper we describe the first 12-month phase of the three-year Care Of Memory Problems in Advanced Stages: Improving Our Knowledge programme which takes place in the UK. We shall use mixed methods to collect fine-grained qualitative and quantitative data on the experiences of people with advanced dementia and their carers as death approaches and in bereavement. Findings will inform the development of a new model of care (intervention) in the next phase of this research (figure 1). This work responds to the UK government initiatives to provide rigorous evidence to improve the experience of people with dementia and their carers around death and in bereavement. ${ }^{9} 1920$ The research has been adopted onto the portfolios of the National Institute for Health Research Clinical Research Network (NIHR CRN) - Primary Care Research Network (PCRN) (Refs. 12621; 12623) and the Dementia and Neurodegenerative Diseases Research Network (DeNDRoN).

\section{METHODS}

Aim

To understand the end-of-life experience of people with dementia and their carers and to generate information on how this could be improved.

\section{Objectives}

1. In people with advanced dementia we will study in depth their clinical symptom burden and health and social care needs, and pathways of care as the end-of-life approaches.

2. In informal family carers to understand in depth their needs and experiences in the period before bereavement and in the months after their loss.

3. To gather views from professional and informal carers on how care could be improved.

\section{Literature update}

We shall begin by updating existing reviews of the international literature on end-of-life interventions for people with advanced dementia and their carers and carer bereavement. ${ }^{5} 122122$

\section{Cohort studies (quantitative data)}

We shall conduct prospective cohort studies of people with advanced dementia and their carers.

\section{People with advanced dementia}

\section{Study population}

With assistance from the PCRN we shall recruit 100 people with advanced dementia, 50 in patient-carer dyads, $70 \%$ residing in care homes and $30 \%$ in their own homes ${ }^{1}$ from 10 boroughs in London, UK. We shall purposively select 10-20 care homes served by general practices (GP) linked to the PCRN that are representative of variations in quality, number of beds and type of ownership.

We will include people over 65 years of age with a clinical diagnosis of dementia (DSM-IV criteria), ${ }^{23}$ at the FAST grade $7 \mathrm{c}$ and above $^{16}$ (unable to walk, all intelligible speech is lost and doubly incontinent). As people with advanced dementia will be unable to give informed consent; we will gain assent from a carer ('personal consultee') or professional consultee who is able to give assent. We will not include those who indicate either verbally or non-verbally that they do not wish to participate, those who are moribund, comatose or where there are clinical concerns that may preclude them being considered for inclusion.

\section{Consent and recruitment procedure for people with advanced dementia}

We have obtained approval from a Research Ethics Committee specialised in considering research on adults 
Figure 1 The Care Of Memory Problems in Advanced Stages: Improving Our Knowledge programme, first phase.

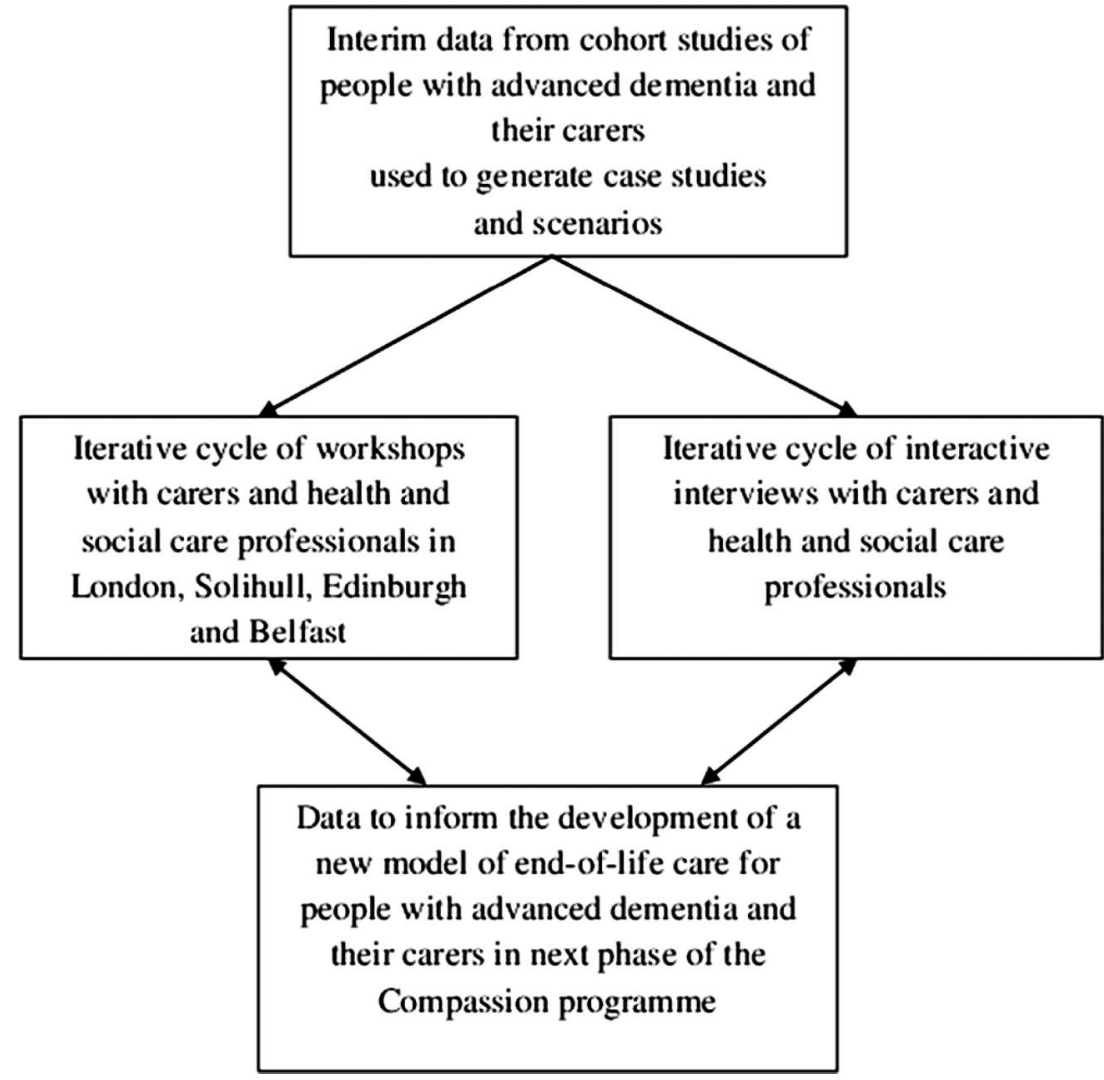

who lack capacity. We shall gain assent from the next-of-kin, unpaid carer or close person to act as a 'personal consultee' according to the Mental Capacity Act (UK) 2005. If no 'personal consultee' is available the research team will nominate a clinician to act in this capacity as a 'professional consultee', defined according to current guidance $^{9}$ as a "professional who is not directly involved in the research or the care of the person with dementia'. To ensure collection of maximum data, consultees will be asked for consent for the research team to access health and social care records across primary and secondary care. We have used similar processes in earlier work funded by the Alzheimer's Society and BUPA Foundation (BePaid grant ref. 131). ${ }^{24}$

\section{Data collection}

We will collect demographic information at baseline including information obtained from GP notes such as medications; acute hospital admissions and the reasons for these; and 'sentinel events', defined as 'new medical conditions that have the potential to lead to a significant change in health status and a shift in the goals of care', for example, pneumonia and hip fracture. ${ }^{17}$ The sources of data are summarised in table 1. Patients will be reviewed every 4 weeks for a maximum of 9 months or until death. We will document the presence of advance directives, care plans and specific requests regarding hospitalisation and resuscitation. ${ }^{9}$

\section{Health economics}

We will calculate costs for the last 6 months of life. We will combine the volume of resource use data obtained for people with advanced dementia and their carers with unit costs data from published sources ${ }^{37} 38$ in order to calculate costs per patient from NHS, personal social services and societal perspectives.

\section{Data analysis}

Interim descriptive analyses will be conducted at 6, 12 and 24 weeks which will inform our understanding of patient outcomes, symptom burden, mortality, patient pathways, case studies and scenarios. After full data collection, we shall present the cohort demographics, the symptoms experienced and calculate symptom rates over the follow-up period (with CIs), taking into account repeated measures. We shall explore the level and nature of unmet needs, describing how comfort and quality of life change over time and differences between those living in care homes and those remaining in their own homes.

\section{Sample size}

Numbers are chosen pragmatically, guided by the literature, ${ }^{17}$ and the anticipated feasibility of recruitment rates and survival times of people at FAST stage 7c. 
Table 1 Summary of assessments for people with advanced dementia

\begin{tabular}{|c|c|c|c|}
\hline Measure/author & Purpose & Source* $^{*}$ & $\begin{array}{l}\text { Time of } \\
\text { assessment }\end{array}$ \\
\hline 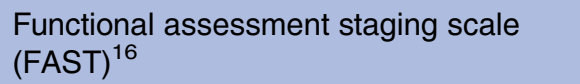 & $\begin{array}{l}\text { Describes a continuum of seven stages of } \\
\text { functional impairment }\end{array}$ & 2 & Baseline \\
\hline $\begin{array}{l}\text { Diagnostic statistical manual of mental } \\
\text { disorders (DSM-1V) }\end{array}$ & Diagnostic criteria for dementia & 2 & Baseline \\
\hline Charlson co-morbidity index ${ }^{25}$ & Calculates severity of chronic comorbidities & 2 & Baseline \\
\hline Waterlow scale 26 & Risk for developing pressure sores & 2,3 & Baseline/monthly \\
\hline Sterling wound assessment scale ${ }^{27}$ & Assesses extent of wound damage & 2,3 & Baseline/monthly \\
\hline $\begin{array}{l}\text { Bedford Alzheimer nursing scale } \\
\text { (BANS) }^{28}\end{array}$ & $\begin{array}{l}\text { Used to stage levels of severe memory } \\
\text { impairment in dementia }\end{array}$ & 2,3 & Baseline/monthly \\
\hline $\begin{array}{l}\text { Confusion assessment measure } \\
(\mathrm{CAM})^{29}\end{array}$ & $\begin{array}{l}\text { Measures confusion and diagnoses delirium and } \\
\text { distinguishes between delirium and dementia }\end{array}$ & $1,2,3,4$ & Baseline/monthly \\
\hline The neuropsychiatric inventory (NPI) ${ }^{30}$ & $\begin{array}{l}\text { Assesses behavioural and psychological } \\
\text { symptoms in dementia }\end{array}$ & $2,3,4$ & Baseline/monthly \\
\hline $\begin{array}{l}\text { Cohen Mansfield agitation inventory } \\
(\mathrm{CMAI})^{31}\end{array}$ & $\begin{array}{l}\text { Measures a range of agitated and potentially } \\
\text { challenging behaviours }\end{array}$ & $2,3,4$ & Baseline/monthly \\
\hline $\begin{array}{l}\text { Pain assessment in advanced dementia } \\
\text { (PAINAD) }^{32}\end{array}$ & $\begin{array}{l}\text { Observational pain tool used to measures pain } \\
\text { during care tasks and at rest }\end{array}$ & 1 & Baseline/monthly \\
\hline $\begin{array}{l}\text { Symptom management at the end of life } \\
\text { in dementia scale (SM-EOLD) })^{33}\end{array}$ & Assesses comfort and pain in the prior 30 days & $1,2,3,4$ & Baseline/monthly \\
\hline $\begin{array}{l}\text { Quality of life in late stage dementia } \\
\text { scale (QUALID) }\end{array}$ & Quality of life in the prior week & 3,4 & Baseline/monthly \\
\hline Painful interventions Scale ${ }^{35}$ & $\begin{array}{l}\text { Lists painful interventions, eg,insertion of } \\
\text { nasogastric tube }\end{array}$ & $2,3,4$ & $\begin{array}{l}\text { Baseline/monthly/ } \\
\text { post death }\end{array}$ \\
\hline $\begin{array}{l}\text { The resource utilisation in dementia } \\
\text { questionnaire (RUD-LITE) }\end{array}$ & $\begin{array}{l}\text { Formal and informal health and social care } \\
\text { resource use }\end{array}$ & $2,3,4$ & $\begin{array}{l}\text { Baseline/monthly/ } \\
\text { post death }\end{array}$ \\
\hline $\begin{array}{l}\text { Client services receipt inventory } \\
(\mathrm{CSRI})^{37}\end{array}$ & As above but more financially based & $2,3,4$ & $\begin{array}{l}\text { Baseline/monthly/ } \\
\text { post death }\end{array}$ \\
\hline $\begin{array}{l}\text { Comfort assessment in dying } \\
\text { (CAD-EOLD) }^{33}\end{array}$ & Comfort assessment in dying with dementia & $2,3,4$ & Death \\
\hline
\end{tabular}

\section{Informal carers}

\section{Study population, consent and recruitment procedures}

We will recruit 50 informal carers of people with advanced dementia, defined as unpaid informal carer (eg, family member or friend in regular contact and who is the next of kin or a 'key decision maker'). They will require English language sufficient to complete the study ratings. We will exclude carers where there are clinical concerns that may preclude them being approached. Carers will be informed that some patient and carer experiences may be used to generate patient pathways, case studies or scenarios. Carers will be invited to take part when approached about considering the participation of their relative of friend in the patient cohort study. They will give informed consent according to usual ethics procedures.

\section{Assessments}

We shall collect data during face-to-face interviews at baseline and every 4 weeks by post or over the telephone. At baseline we shall collect demographic data including relationship to the person with dementia, the number of years spent caring and any other caring responsibilities. All other carer assessments are summarised in table 2 .

\section{Data collection in bereavement}

If the patient dies, carers will be interviewed in a place of their choice at 7 months post-bereavement to consider complicated grief which requires 6 months of symptoms, using the Inventory for Complicated Grief. ${ }^{43}$ If complicated grief is identified, participants will be supported to access appropriate services. We shall undertake interviews which we will analyse using qualitative methods, to gain a deeper understanding of the circumstances surrounding the death and the views of the carer on which aspects of care were or were not satisfactory.

\section{Data analysis}

We shall describe the demographic characteristics of carers and their well-being at study entry and explore descriptively the trajectory of carer well-being in tandem with patient data and how this changes if the person with dementia dies. Data will include carer outcomes as listed in table 2. 
Table 2 Summary of assessments for carers

\begin{tabular}{|c|c|c|c|}
\hline Measure/author & Purpose & Source $^{*}$ & Time of assessment \\
\hline Brief COPE ${ }^{39}$ & Measures coping strategies used & 4 & Baseline \\
\hline Zarit burden interview ${ }^{40}$ & $\begin{array}{l}\text { Reflects feelings about caring, measures } \\
\text { carer burden }\end{array}$ & 4 & Baseline/monthly \\
\hline $\begin{array}{l}\text { Short form } 12 \text { health survey } \\
\text { questionnaire (SF-12) }\end{array}$ & $\begin{array}{l}\text { Measures health related quality of life over } \\
\text { eight domains }\end{array}$ & 4 & $\begin{array}{l}\text { Baseline/monthly/2 and } \\
7 \text { months postdeath }\end{array}$ \\
\hline $\begin{array}{l}\text { Hospital anxiety and depression scale } \\
(\text { HADS) })^{42}\end{array}$ & Anxiety and depression & 4 & $\begin{array}{l}\text { Baseline/monthly/2 and } \\
7 \text { months postdeath }\end{array}$ \\
\hline $\begin{array}{l}\text { Inventory of complicated grief (ICG) } \\
\text { pre-loss } 43\end{array}$ & $\begin{array}{l}\text { Assesses prevalence and severity of } \\
\text { carers' anticipatory grief }\end{array}$ & 4 & Baseline \\
\hline $\begin{array}{l}\text { Inventory of complicated grief (ICG) } \\
\text { pre-loss (ICG-R) postloss }{ }^{43}\end{array}$ & Identifies symptoms of complicated grief & 4 & 2 and 7 months post death \\
\hline $\begin{array}{l}\text { The resource utilization in dementia } \\
\text { questionnaire (RUD-LITE) }\end{array}$ & $\begin{array}{l}\text { Formal and informal health and social } \\
\text { care resource use }\end{array}$ & 4 & $\begin{array}{l}\text { Baseline/monthly/2 months } \\
\text { postdeath }\end{array}$ \\
\hline $\begin{array}{l}\text { Client services receipt Inventory } \\
(\mathrm{CSRI})^{37}\end{array}$ & As above but more financially based & 4 & $\begin{array}{l}\text { Baseline/monthly/2 months } \\
\text { postdeath }\end{array}$ \\
\hline $\begin{array}{l}\text { Satisfaction with care at the end of life } \\
(\text { SWC/CAD-EOLD })^{33}\end{array}$ & $\begin{array}{l}\text { Assesses the carers' level of satisfaction } \\
\text { with care in advanced dementia }\end{array}$ & 4 & $\begin{array}{l}\text { Baseline/monthly/2 months } \\
\text { postdeath }\end{array}$ \\
\hline
\end{tabular}

\section{Workshops and interactive interviews (qualitative data)}

We shall hold an iterative cycle of workshops and interactive interviews with carers and healthcare professionals to generate hypotheses on how end-of-life care for people with advanced dementia and their carers might be improved. Case studies, scenarios and examples of patient pathways and carer experiences informed by data from the cohort studies will be used to generate discussions. Data generated may challenge existing ideas and will lead to the emergence of new theories for further exploration. Themes explored at the workshops will be revised as the study progresses. ${ }^{44} 45$

\section{Workshops with health and social care professionals Participants}

We shall purposively recruit participants from a range of statutory and private providers, and professionals from a variety of disciplines for example, general practitioners, district nurses, speech and language therapists, palliative care specialist nurse/doctors, psychiatric nurses, psychiatrists, geriatricians, Admiral nurses, ambulance staff, social workers, care home staff and commissioners. Potential participants will be sent a study information sheet stating that they have been identified as a person who currently or previously has shown interest in providing end of life are for people with severe memory problems and their carers. They will give informed consent before taking part. Workshops will be facilitated by two members of the research team. A total of 12 workshops will take place over 6 months each with a maximum of 10 participants.

\section{Location}

To understand and consider regional variation in resources, services, needs and policy we shall purposively select providers and commissioners of health and social care in primary, secondary and tertiary settings from four areas of the UK (London, Belfast, Edinburgh and Solihull).

\section{Workshops with carers}

Workshops will be conducted with past and present informal carers of people with advanced dementia.

\section{Recruitment and consent}

To ensure our population is representative, carers of people with advanced dementia will be recruited from across London. Seventy per cent of people with advanced dementia reside in care homes and $30 \%$ at home and we shall stratify our sample to reflect this. Carers will be acting in an unpaid informal role and will be identified by GP linked to the PCRN or by care home managers.

\section{Inclusion criteria}

Those eligible for inclusion will be main informal carers of people with advanced dementia (eg, family member or friend in regular contact who is either next of kin or a 'key decision maker') with English language skills sufficient to participate in workshops or interactive interviews. We shall not include carers under the age of 16 or those for whom there are clinical concerns that may preclude them from being approached such as severe physical or mental illness or lack of capacity to give informed consent.

\section{Workshops with people with early stage dementia}

We shall conduct up to two workshops with people with early dementia. 


\section{Recruitment and consent}

People with early stage dementia who have a Mini Mental State Examination ${ }^{46}$ of 20 and above and who are under the care of a specialist memory clinic will be recruited to participate in workshops using the North Thames DeNDRoN research register (DemReg), a database of patients from memory clinics and old age psychiatry services who have given signed consent to be approached to participate in research studies. We will not include people if there are clinical concerns precluding them from being approached, those who lack capacity to consent at the outset and those who are unable to communicate in English to a degree whereby they would be unable to participate in the workshops. Using a fourpoint framework (Mental Capacity Act, 2005; p45), the researcher will make an independent assessment of the capacity of the person with dementia at the point of recruitment. To participate, they will be able to: (1) understand (relevant) information about the decision to be made; (2) retain this; (3) use or weigh the information as part of the decision-making process and (4) communicate their decision.

\section{Data analysis}

One researcher will take detailed field notes during the workshops to record the setting, the activities that took place, who participated and their role in the activities and exact quotations or close approximations of important comments made. We shall use flip-charts to record key elements of discussions. All data will be entered into a qualitative software programme, Atlas-ti and analysed for thematic content emphasising emerging hypotheses on how care may be improved.

\section{Interviews with carers and healthcare professionals}

Unstructured interactive interviews are appropriate for understanding complex issues that are relevant to healthcare settings. ${ }^{47}$ Such interviews provide participants considerable control over the interview process ${ }^{48}$ and are similar to guided conversations. ${ }^{47}$ We shall conduct individual interactive interviews with carers and healthcare professionals. As with the workshops, discussions will be facilitated using case studies and scenarios of patient and carer experience. These interactive interviews take an account of the natural interaction between researcher and participant through which each unconsciously considers underlying hypotheses and contextualises responses. Our approach will be underpinned by critical realism attempting to uncover influential processes, policies, perceptions and events that often determine health and social care outcomes. A maximum of 10 interviews will occur only in London at 8, 14 and 30 weeks from the start of the cohort studies, giving 30 interviews with healthcare professionals and 30 interviews with carers. Themes explored during the interviews will be revised as the study progresses.

\section{Health and social care professionals}

As with the workshops, interviews will be conducted with a wide range of health and social care professionals to provide an overall perspective. Topics may include discussion about health and social care professionals' roles and responsibilities for patients with advanced dementia. We shall explore what they perceive as the good and bad things about their role and their relationships with other professionals in the field. This will lead to discussions about what an ideal model of care would look like, how this compares with what actually happens now and how improvements might be achieved. We shall also explore how people respond to things that go wrong in everyday practice.

\section{Carers}

Interviews will be conducted with current and past carers of people with advanced dementia. We shall explore in depth the complex and potentially contested layers of patient and carer experience and how changes in the management of advanced dementia could improve care pathways. More specifically, topics may include illness beliefs and the illness journey, carer needs along the journey, communication and information needs, the carers' role in decision making, attitudes towards end-of-life care and palliative care principles.

\section{Data analysis}

Interviews will be audio-taped, transcribed verbatim and entered into Atlas-ti. The transcripts will be combined with field notes, memos and any relevant policy documents to reflect upon our initial hypotheses. Thus, we shall identify and clarify the barriers to the provision of good health and social care by revealing underlying, often unseen, factors and processes. With the support of diagrams and charts, the analysis will detail and explain the complex linkages between actors and agencies, structural and cultural factors. We shall explore the best way of providing a clear description of key issues that can be fully appreciated by a range of professionals and carers. For example, while we shall undertake a thematic analysis of the data we shall also seek to describe particular barriers through a revision of the case studies and vignettes in a way that accommodates the new explanatory information in an accessible way. While we shall look for patterns or commonalities of behaviour and events we shall also examine and explain irregular cases. The analysis and findings will allow us to build a comprehensive set of recommendations to be incorporated within the development of our intervention. This will also stand alone as a significant contribution to the evidence base of end-of-life care.

\section{Patient and public involvement}

We are experienced in involving people with dementia and their relatives in research. ${ }^{49} 50$ We shall involve people with early dementia, carers are included in 
workshops across the UK and an expert by experience joins our steering group.

\section{Expert steering group}

An expert steering group will meet three times per year. The group is composed of stakeholders with expertise in end-of-life care and dementia including service providers and policy makers from statutory and voluntary bodies. The group will monitor the progress of the research, review interim findings and ensure the appropriate media and policy profile of the work as it unfolds.

\section{ETHICS AND DISSEMINATION}

We have obtained approval from NHS ethical committees through IRAS for studies involving people with dementia and carers (cohort studies REC ref. 12/EE/ 0003; workshops and interactive interviews REC ref. 12/ LO/0346), and from university ethics committees for work with health and social care professionals (REC ref. 3578/001).

We shall prepare documents for dissemination by end-of-life and dementia care organisations such as Marie Curie Cancer Care, BUPA, Dementia UK, The Alzheimer's Society, National Council for Palliative Care, National End of Life Care programme and the government special advisor for dementia including detailed reports, scientific presentations at conferences and papers for peer reviewed journals, and publicise our findings on the Marie Curie website. A summary will be provided to all participants who would like to receive this.

\section{DISCUSSION}

Combining the quantitative and qualitative data from this study will enable us to gain a greater and more detailed understanding of the clinical and social care needs of people with advanced dementia and their carers as they face death, and for carers into bereavement. Data will inform explanations of the barriers to good care and generate hypotheses on how these can be challenged in new ways to provide innovative solutions. We shall be able to report in detail on the clinical symptom burden of people with advanced dementia living in care homes and their own homes and on their survival. We will gain an in-depth understanding of the complex interactions between the patient experience of care, the involvement of carers and the health and social care systems in which care is delivered and received. ${ }^{45}$ Our mixed methods in-depth approach will enable us to consider factors that might affect the implementation of an intervention from the outset. ${ }^{51} 52$

\section{Further work}

We have received funding from Marie Curie Cancer Care for subsequent research in which we shall use these data to inform the development of a complex intervention. We shall collect data on its acceptability, feasibility and potential costs by piloting the intervention in one healthcare economy before proceeding to further testing in an exploratory randomised controlled trial.

\section{Strengths and limitations}

While there have been some prospective cohort studies in other countries ${ }^{53}$ to our knowledge our quantitative data will be the first of this nature to be collected in the UK population. We are attempting to ensure that we recruit a representative sample of people with advanced dementia and their family carers, and have used professional consultees so that people with advanced dementia who may not have a relative to assent for them can still be included in research. Using qualitative research methods will provide a richness and background to this quantitative data, allowing it to be better placed in the context of a complex health and social care system. While dementia is a terminal disease in its own right, our research does not address the palliative and end-of-life care needs of those dying at earlier stages of dementia from other conditions.

\section{CONCLUSION}

We know that as the numbers dying with dementia continue to increase, this programme will be of importance to health and social care service planners. The analysis and findings will allow us to build a comprehensive set of recommendations to be incorporated within the development of our complex intervention. Policy in this field is rapidly developing and we shall report findings as soon as they are available to ensure that the insights we gain enter the public domain as quickly as possible.

Contributors LJ and ELS devised the programme of research and secured the funding; LJ, ELS, SS and JH wrote the study protocols. LJ, ELS and JH drafted the manuscript. All authors critically revised the manuscript for important intellectual content. All authors read and approved the final manuscript.

Funding This programme of research has been fully funded by Marie Curie Cancer Care (grant ref. MCCC-FPR-11-U) through a process administered in partnership with Cancer Research UK.

Competing interests None.

Ethics approval NHS Ethics Comittees and UCL Ethics Committee.

Provenance and peer review Not commissioned; internally peer reviewed.

\section{REFERENCES}

1. Knapp M, Prince M, Albanese E, et al. Dementia UK, Alzheimer's Society, London. 2007. http://www.alzheimers.org.uk/site/scripts/ download.php?filelD=2 (accessed Oct 2012).

2. Brayne C, Gao L, Dewey M, et al. Dementia before death in ageing societies-the promise of prevention and the reality. PLoS Med 2006;10:e397.

3. Dewey ME, Saz P. Dementia, cognitive impairment and mortality in persons aged 65 and over living in the community: a systematic review of the literature. Int J Geriatr Psychiatry 2001;16:751-61.

4. Sampson EL, Bulpitt CJ, Fletcher AE. Survival of community-dwelling older people: the effect of cognitive impairment and social engagement. J Am Geriatr Soc 2009;57:985-91.

5. Sampson EL, Gould V, Lee D, et al. Differences in care received by patients with and without dementia who died during acute hospital admission: a retrospective case note study. Age Ageing 2006;35:187-9. 
6. Davies E, Higginson IJ. Better palliative care for older people. Copenhagen: World Health Organisation Europe, 2004. http://www. euro.who.int/ data/assets/pdf_file/0009/98235/E82933.pdf (accessed Oct 2012).

7. Sanders S, Swails P. Caring for individuals with end-stage dementia at the end of life. Dementia 2009;8:117-38.

8. McCarty CE, Volicer L. Hospice access for individuals with dementia. Am J Alzheimers Dis Other Demen 2009;24:476-85.

9. Department of Health. End of life care strategy: promoting high quality care for all adults at the end of life. London: Department of Health, 2008.

10. Bourne J. Improving services and support for people with dementia. London: National Audit Office, 2007. http://www.nao.org.uk/ publications/0607/support_for_people_with_dement.aspx (accessed Oct 2012)

11. Sampson EL, Ritchie CW, Lai R, et al. A systematic review of the scientific evidence for the efficacy of a palliative care approach in advanced dementia. Int Psychogeriatr 2005;17:31-40.

12. Goodman C, Evans C, Wilcock J, et al. End of life care for community dwelling older people with dementia: an integrated review. Int J Geriatr Psychiatry 2010;25:329-37.

13. Jordan $\mathrm{A}$. Extending palliative care to people with dementia. $\mathrm{Br} \mathrm{J}$ Hosp Med (Lond) 2010;71:31-5.

14. Campbell ML, Dove-Medows E, Walch J, et al. The impact of multidisciplinary educational intervention to reduce PEG tube placement in patients with terminal-stage dementia: a translation of research into practice. J Palliat Med 2011;14:1017-21.

15. Hanson LC, Carey TS, Caprio AJ, et al. Improving decision-making for feeding options in advanced dementia: a randomized, controlled trial. J Am Geriat Soc 2011:59:2009-16.

16. Reisberg B. Functional assessment staging (FAST). Psychopharmacol Bull 1988;24:653-9.

17. Mitchell SL, Teno JM, Kiely DK, et al. The clinical course of advanced dementia. N Engl J Med 2009;361:1529-38.

18. McCarthy M, Addington-Hall J, Altmann D. The experience of dying with dementia: a retrospective study. Int J Geriatr Psychiatry 1997; 12:404-9.

19. The coalition. Our programme for government. London: Cabinet Office, 2010. http://www.cabinetoffice.gov.uk/news/ coalition-our-programme-government (accessed Oct 2012).

20. Department of Health. Prime Minister's challenge on dementia. Delivering major improvements in dementia care and research by 2015. London: Department of Health, 2012. http://www.dh.gov.uk/ health/2012/03/pm-dementia-challenge/ (accessed Oct 2012).

21. van der Steen JT. Dying with dementia: what we know after more than a decade of research. J Alzheimers Dis 2010;22:37-55.

22. Chan D, Livingston $\mathrm{G}$, Jones $\mathrm{L}$, et al. Grief reactions in dementia caregivers: a systematic review. Int $J$ Geriatr Psychiatry Published online first: 8 March 2012. doi: 10.1002/gps.3795.

23. American Psychiatric Association. Diagnostic and statistical manual of mental disorders (DSM-1V). Washington, DC: American Psychiatric Association, 1994.

24. Scott S, Jones L, Blanchard MR, et al. Study protocol: the Behaviour and Pain in Dementia Study (BePAID). BMC Geriatr 2011;16:61. http:// www.biomedcentral.com/1471-2318/11/61 (accessed Oct 2012).

25. Charlson ME. Studies of prognosis: progress and pitfalls. J Gen Intern Med 1987;2:359-61.

26. Waterlow J. Pressure sores: a risk assessment card. Nurs Times 1985;81:49-55.

27. Reid J, Morison M. Classification of pressure sore severity. Nurs Times 1994;90:46-50.

28. Volicer L, Hurley AC, Lathi DC, et al. Measurement of severity in advanced Alzheimer's disease, J Gerontol 1994:49:M223-6.

29. Inouye SK, van Dyck CH, Alessi CA, et al. Clarifying confusion: the confusion assessment method. A new method for detection of delirium. Ann Intern Med 1990;113:941-8.
30. Cummings JL, Mega M, Gray K, et al. The Neuropsychiatric Inventory: comprehensive assessment of psychopathology in dementia. Neurology 1994;44:2308-14.

31. Cohen-Mansfield J, Marx MS, Rosenthal AS. A description of agitation in a nursing home. J Gerontol 1989;44:M77-84.

32. Zwakhalen SM, Hamers JP, bu-Saad $\mathrm{HH}$, et al. Pain in elderly people with severe dementia: a systematic review of behavioural pain assessment tools. BMC Geriatr 2006;6:3.

33. Kiely DK, Volicer L, Teno J, et al. The validity and reliability of scales for the evaluation of end-of-life care in advanced dementia. Alzheimer Dis Assoc Disord 2006;20:176-81.

34. Weiner MF, Martin-Cook K, Svetlik DA, et al. The quality of life in late-stage dementia (QUALID) scale. J Am Med Dir Assoc 2000;1:114-16.

35. Morrison RS, Ahronheim JC, Morrison GR, et al. Pain and discomfort associated with common hospital procedures and experiences. J Pain Symptom Manage 1998;15:91-101.

36. Wimo A, Winblad B. Resource utilisation in dementia: RUD Lite. Brain Aging 2003;3:48-59.

37. Beecham J, Knapp M. Costing psychiatric interventions. In: Thornicroft G, ed. Measuring mental health needs. London: Gaskell, 2001:200-24.

38. Netten A, Curtis L. Unit costs of health and social care. Canterbury: Personal Social Services and Research Unit, 2005.

39. Carver CS. You want to measure coping but your protocol's too long: consider the brief COPE. Int J Behav Med 1997;4:92-100.

40. Zarit SH, Reever KE, Bach-Peterson J. Relatives of the impaired elderly: correlates of feelings of burden. Gerontologist 1980;20:649-55.

41. Ware J Jr, Kosinski M, Keller SD. A 12-item short-form health survey: construction of scales and preliminary tests of reliability and validity. Med Care 1996;34:220-33.

42. Zigmond AS, Snaith RP. The hospital anxiety and depression scale. Acta Psychiatr Scand 1983;67:361-70.

43. Prigerson HG, Maciejewski PK, Reynolds CF III, et al. Inventory of Complicated Grief: a scale to measure maladaptive symptoms of loss. Psychiatry Res 1995;59:65-79.

44. Sampson EL, Mandal U, Holman A, et al. Improving end of life care for people with dementia: a rapid participatory appraisal. BMJ Support Palliat Care 2012;2:108-14.

45. Harrison-Dening K, Greenish W, Jones L, et al. Barriers to providing end of life care for people with dementia: a whole-system qualitative study. BMJ Support and Palliat Care 2012;2:103-7.

46. Folstein MF, Folstein SE, McHugh PR. 'Mini-mental state'. A practical method for grading the cognitive state of patients for the clinician. J Psychiatr Res 1975;12:189-98.

47. DiCicc0-Bloom B, Crabtree BF. The qualitative research interview. Med Educ 2006;40:314-21.

48. Corbin J, Morse JM. The unstructured interactive interview: issues of reciprocity and risks when dealing with sensitive topics. Qual Inquiry 2003;9:335-54.

49. Livingston $\mathrm{G}$, Leavey $\mathrm{G}$, Manela $\mathrm{M}$, et al. Making decisions for people with dementia who lack capacity: qualitative study of family carers in UK. BMJ 2010;341:c4184.

50. Dartington T, Coupe K, Malloy L, et al. Dying from dementia-a patient's journey. BMJ 2008;337:a1712.

51. Campbell M, Fitzpatrick R, Haines A, et al. Framework for design and evaluation of complex interventions to improve health. $B M J$ 2000;321:694-6.

52. Craig $P$, Dieppe $P$, Macintyre $S$, et al. Developing and evaluating complex interventions: the new Medical Research Council guidance. BMJ 2008;337:979-83.

53. Mitchell SL, Kiely DK, Jones RN, et al. Advanced dementia in the nursing home: the CASCADE study. Alzheimer Dis Assoc Disord 2006;20:166-75. 